\title{
Discussion on Curative Effect and Safety of Small-dosage Alteplase Combined with Urokinase in Treatment of Acute Pulmonary Embolism
}

\author{
Gongchun Fan ${ }^{1}$, Xingguo Peng ${ }^{2, *}$, Kun Zhang ${ }^{3}$ \\ ${ }^{1}$ Department of Respiratory Medicine, Renmin Hospital, Hubei University of Medicine, Shiyan \\ Renmin Hospital, Shiyan, Hubei Province, China,442000 \\ ${ }^{2}$ Center of Emergency and Critical Care, Renmin Hospital, Hubei University of Medicine, \\ Shiyan Renmin Hospital, Shiyan, Hubei Province, China,442000 \\ 3The first clinical college of Wuhan University, Wuhan, Hubei Province, China, 430000 \\ "Corresponding author: hubeiwenbo@163.com \\ Address: Chaoyang District Road No. 39, Shiyan City, Hubei Prov, P.R.China, 442000
}

Keywords: acute pulmonary embolism; alteplase; urokinase; curative effect; safety

\begin{abstract}
Objective: to discuss curative effect and safety of small-dosage alteplase combined with urokinase in treatment of acute pulmonary embolism. Method: the inclusion time bucket of the objects of this study was from January 2017 to March 2018. Random number table was used to choose 64 patients with acute pulmonary embolism received in our hospital. Then, single blind method was used to classify them into control group and observation group. Each group included 32 cases. The control group received pure urokinase treatment, while the observation group received the treatment by small-dosage alteplase combined with urokinase. The clinical data of both groups were sorted, compared and analyzed in detail. Results: the improvement degree of pulmonary artery systolic pressure and arterial partial pressure of oxygen in the observation group was better than that in the control group. Total effective rate of clinical treatment was 93.75\%, higher than that of control group (75.00\%), $\mathrm{P}<0.05$. Bleeding rate of observation group was $6.25 \%$, while the bleeding rate of control group was 3.13\%, without comparison difference, $\mathrm{P}>0.05$. Conclusion: small-dosage alteplase combined with urokinase has the better clinical effect and higher safety for the patients with acute pulmonary embolism.
\end{abstract}

Since endogenous and exogenous emboli of the patients with acute pulmonary embolism block pulmonary artery and its branches, pulmonary circulation disorder occurs. Any carelessness will make patients lose life ${ }^{[1]}$. At present, many drugs can be used to treat pulmonary artery embolism clinically, but the clinical effect differs. To further improve clinical treatment effect of pulmonary artery embolism, the objects were classified into two groups, and different treatment schemes were provided for them to discuss clinical effect and safety of small-dosage alteplase combined with urokinase in the treatment of acute pulmonary embolism. 


\section{Data and Method}

\subsection{General data}

In this study, 64 patients with acute pulmonary embolism who were received in our hospital from January 2017 to March 2018 were chosen as the objects with random number table method. Then, single blind method was used to classify the objects into control group and observation group. Each group included 32 cases. In the control group, the proportion of male and female patients was $18: 14$, and the age was $23-80$, with the average age of $53.74 \pm 5.07$. The duration from onset to acceptance of treatment was 6h-13d, with the average time of $(10.50 \pm 1.30)$ h. In the observation group, the proportion of male and female patients was 19:13, and the age was 25-81, with the average age of 53.91 \pm 5.55 . The duration from onset to acceptance of treatment was $7 \mathrm{~h}-12 \mathrm{~d}$, with the average time of $(10.43 \pm 1.07) \mathrm{h}$. The clinical data of both groups were entered in SPSS 20.0 statistical software, and the analysis results had no difference, $\mathrm{P}>0.05$. The research content conformed to medical ethics and also gained the approval of Ethics Committee of the hospital.

Inclusion criteria: (1) the disease of patients was verified by clinical pathology and imageology, with typical clinical symptoms, including chest pain, dyspnea or polypnea, syncope, hemoptysis and hyoxemia etc.; (2) accompanied by shock, systolic pressure $\leq 90 \mathrm{mmHg}$, duration $>15 \mathrm{~min}$, pulmonary artery high pressure and (or) right cardiac insufficiency; (3) the disease time did not exceed 14d; the family members knew the research content and signed the informed consent form.

Exclusion criteria: (1) combined with other major organ diseases or functional insufficiency; (2) cerebral apoplexy and intracranial hemorrhage occurred within 3d after the onset; (3) retinal hemorrhage; hemorrhage in other parts; blood platelet count did not exceed $100 \times 10^{9} / \mathrm{L}$; (4) incomplete clinical data; those who could not cooperate with clinical treatment and research.

\subsection{Method}

Observation group: thrombolytic therapy was implemented for the patients in the first day when the patients were hospitalized. 20mg alteplase (S20110051, Boehringer Ingelheim ) and 600,000U urokinase (GYZZ H20113006, Techpool Bio-Pharma Co., Ltd.) were chosen. From the second day to the fifth day, 600,000U urokinase was given every day.

Control group: 40,000U/kg urokinase was given for the patients in the first day when the patients were hospitalized, and was intravenously injected within 30min. Later, 1,200,000U thrombolytic therapy was carried out. From the second day to the fifth day, 600,000U urokinase was given every day.

After thrombolysis was completed in the first day of treatment, patients' activated partial thromboplastin time (APTT) was closely monitored. If the value is lower than the normal value, low molecular heparin should be given for the patients $(0.1 \mathrm{ml} / 10 \mathrm{~kg})$ by subcutaneous injection. The drug was injected for the patients at the interval of $12 \mathrm{~h}$. After continuous injection of $5 \mathrm{~d}$, warfarin started to be given. If international normalized ratio of prothrombin time is2-3 times higher than the normal value, low molecular heparin should stop giving at once. The patients should take warfarin for 12 consecutive months ${ }^{[2]}$.

\subsection{Evaluation criteria of curative effect}

Clinical effect judgment criterion evaluation is referred to in Literature [3]: patients' clinical 
symptoms are improved significantly or disappear, and pulmonary artery embolism has been improved obviously or recovered, promoting the curative effect is significant. Patients' clinical symptoms are improved slightly or relieved, and degree of pulmonary artery stenosis does not exceed $50 \%$ or thrombus has been dissolved, promoting the curative effect is good. Patients' clinical symptoms do not change obviously, and degree of pulmonary artery thrombolysis does not exceed $25 \%$, or no obvious change is seen, promoting the treatment fails. Patients' clinical symptoms are exacerbated, and the coloboma number of pulmonary artery embolism segment increases, promoting disease exacerbation. Obvious effect + effect $=$ total effect, expressed with \%.

\subsection{Statistical method}

SPSS 20.0 statistical software was used to analyze clinical data. Measurement data were expressed with $x \pm s$, and tested with $t$ test. Enumeration data were expressed with \%, and tested with chi-square test. Statistical software analysis showed $\mathrm{P}<0.05$, with statistical significance.

\section{Results}

\subsection{Comparison of pulmonary embolism improvement before and after treatment}

Before the treatment, the comparison of pulmonary arterial systolic pressure and arterial partial pressure of oxygen in both groups had no difference, $\mathrm{P}>0.05$. After the treatment, pulmonary arterial systolic pressure and arterial partial pressure of oxygen in both groups were not improved, but the improvement degree of pulmonary arterial systolic pressure and arterial partial pressure of oxygen in the observation group was better than that in the control group, $\mathrm{P}$ $<0.05$, as shown in Table 1 .

Table 1 Pulmonary embolism improvement of both groups before and after treatment ( $x \pm s$, $\mathrm{mmHg}$ )

\begin{tabular}{ccccccc}
\hline Group & \multicolumn{2}{c}{ Pulmonary arterial systolic pressure } & \multicolumn{3}{c}{ Arterial partial pressure of oxygen } \\
\cline { 2 - 7 } & $\begin{array}{c}\text { Treatment } \\
\text { for 1d }\end{array}$ & $\begin{array}{c}\text { Treatment } \\
\text { for 3d }\end{array}$ & $\begin{array}{c}\text { Treatment } \\
\text { for 5d }\end{array}$ & $\begin{array}{c}\text { Treatment } \\
\text { for 1d }\end{array}$ & $\begin{array}{c}\text { Treatment } \\
\text { for 3d }\end{array}$ & $\begin{array}{c}\text { Treatment } \\
\text { for 5d }\end{array}$ \\
\hline $\begin{array}{c}\text { Observation } \\
\text { group }\end{array}$ & $41.19 \pm 9.07$ & $33.95 \pm 8.64$ & $30.71 \pm 6.78$ & $83.90 \pm 7.98$ & $86.42 \pm 8.05$ & $89.97 \pm 9.50$ \\
$\begin{array}{c}\text { Control } \\
\text { group }\end{array}$ & $55.63 \pm 9.15$ & $48.15 \pm 9.02$ & $45.35 \pm 6.85$ & $69.36 \pm 8.02$ & $72.15 \pm 8.37$ & $75.04 \pm 9.32$ \\
t value & 6.3402 & 6.4311 & 6.6304 & 7.2700 & 6.9512 & 6.3461 \\
P value & 0.0467 & 0.0416 & 0.0409 & 0.0365 & 0.0412 & 0.0438 \\
\hline
\end{tabular}

\subsection{Comparison of clinical treatment effect}

Total effective rate of observation group was 93.75\%, higher than that of control group (75.00\%), $\mathrm{P}<0.05$, as shown in Table 2 . 
Table 2 Comparison of clinical treatment effect $(\%, \mathrm{n})$

\begin{tabular}{|c|c|c|c|c|c|c|}
\hline Group & No. & $\begin{array}{c}\text { Significant } \\
\text { effect }\end{array}$ & Effective & Ineffective & Exacerbation & Total effective rate (\%) \\
\hline $\begin{array}{l}\text { Observation } \\
\text { group }\end{array}$ & 32 & 21 & 9 & 2 & 0 & $30(93.75 \%)$ \\
\hline Control group & 32 & 11 & 13 & 7 & 1 & 24(75.00\%) \\
\hline $\mathrm{X}^{2}$ value & - & - & - & - & - & 4.2267 \\
\hline$P$ value & - & - & - & - & - & 0.0389 \\
\hline
\end{tabular}

\subsection{Comparison of hemorrhage rate}

Hemorrhage occurred to 2 patients in the observation group (6.25\%), while hemorrhage occurred to 1 patient in the control group (3.13\%). Hemorrhage rate of observation group was $6.25 \%$, while that of control group was $3.13 \%$, without comparison difference, $X^{2}=0.3497$, $\mathrm{P}=0.5543>0.05$.

\section{Discussion}

For the patients with pulmonary artery embolism, thrombus blocked their pulmonary artery and branches, thus leading to continuous rise of pulmonary circulation resistance. As a result, pulmonary artery pressure continued to rise, and the load of right ventricle expanded, thus leading to right cardiac insufficiency or dysfunction. After embolism, returned blood volume of patients decreased, thus cardiac output reduced and blood pressure dropped. As time went on, patients' life safety would be threatened ${ }^{[4-6]}$. Niu Hongxia et al. ${ }^{[7]}$ indicated that, the state of pulmonary embolism is dangerous, pathogenesis is complex and clinical manifestations are not typical, so misdiagnosis and missed diagnosis cannot be avoided. The fatality rate can reach 20\%-30\%. Thus, seeking a scientific and effective treatment scheme has the favorable reference value for clinical treatment of acute pulmonary embolism.

At present, thrombolytic therapy is often used to treat pulmonary embolism. This method can achieve certain effect, and it is easy and safe to operate. However, the common thrombolytic drug urokinase can easily cause Extensive fibrinogen consumption, and patients may thus bleed ${ }^{[8]}$. Alteplase is a plasminogen activating agent, and has very excellent specificity. It can be combined with fibrous protein in the body to active plasminogen and transform it into plasmin. When plasmin flows to the thrombus with blood, the obvious thrombolysis effect can be seen. The drug can work rapidly. Except rapid thrombus dissolution, it can also promote improvement and recovery of lung tissue reperfusion and obviously improve right cardiac insufficiency ${ }^{[9]}$. Multiple research results show that, large-dosage alteplase can increase patients' hemorrhage rate ${ }^{[10]}$. Thus, the patients in the observation group were treated by small-dosage alteplase $(20 \mathrm{mg})$ combined with urokinase. The results showed that, pulmonary embolism improvement of the observation group was significant. The improvement degree of pulmonary arterial systolic pressure and arterial partial pressure of oxygen in the observation group was better than that in the control group. The total effective rate of clinical treatment in the observation group was higher than that in the control group (75.00\%). Only 6.25\% patients bled. Thus, small-dosage alteplase combined with urokinase has obvious effect. It can fully improve the disease, and well control bleeding risk.

In conclusion, small-dosage alteplase combined with urokinase has high clinical effect in the treatment of acute pulmonary embolism. Besides, it will not increase hemorrhage rate, and it is safe. 


\section{References}

[1] Zhao T, Ni J , Hu X, Wang Y, Du X .The Efficacy and Safety of Intermittent Low-Dose Urokinase Thrombolysis for the Treatment of Senile Acute Intermediate-High-Risk Pulmonary Embolism: A Pilot Trial.Clinical and applied thrombosis/hemostasis : official journal of the International Academy of Clinical and Applied Thrombosis/Hemostasis 2018 Oct; 24 (7):1067-1072.

[2] Willis SL , Welch TS , Scally JP , Bartoszek MW.Impending paradoxical embolism presenting as a pulmonary embolism, transient ischemic attack, and myocardial infarction.Chest 2007 Oct; 132 (4 ):1358-60.

[3] Sullenberger LE , Pamplin JC, Hnatiuk OW .Deep venous thrombosis and pulmonary embolism. Part 1. Initial treatment: usually a low-molecular-weight heparin.Prescrire Int. 2013 Apr;22(137):99-101, 103-4.

[4] Khor YH, Smith R, McDonald CF.Suboptimal management of unfractionated heparin compared with low-molecular-weight heparinin the management of pulmonary embolism.Intern Med J. 2014 Apr;44(4):339-44.

[5] Kleinjan A1, Hutten BA, Di Nisio M, Büller HR, Kamphuisen PW..Anticoagulant treatment of cancer patients with pulmonary embolism in the real world. Actual useof low-molecular-weight heparin in cancer.Neth $J$ Med. 2014 Nov;72(9):467-72.

[6] Mohan PP1, Manov JJ1, Contreras F2, Langston ME1, Doshi MH1, Narayanan G1.Ultrasound-Assisted Catheter-Directed Thrombolysis for Submassive Pulmonary Embolism.Vasc Endovascular Surg. 2018 Apr;52(3):195-201.

[7] Subramanian M1, Ramadurai S1, Arthur P1, Gopalan S1.Hypoxia as an independent predictor of adverse outcomes in pulmonary embolism.Asian Cardiovasc Thorac Ann. 2018 Jan;26(1):38-43.

[8] Zhang S1, Zhai Z2, Yang Y1, Zhu J3, Gong J1, Xie W1, Kuang T1, Wang C4.Long-term treatment with low-molecular-weight heparin prolonged the survival time for acutepulmonary embolism patients concurrent with malignancy: an observational analysis from a long-term follow-up study.Thromb Res. 2015 Apr;135(4):582-7.

[9] Dong J1, Wang J2, Feng Y2, Qi LP3, Fang H1, Wang GD1, Wu ZQ4, Wang HZ1, Yang Y2, Li Q1.Effect of low molecular weight heparin on venous thromboembolism disease in thoracotomy patients with cancer.J Thorac Dis. 2018 Mar;10(3):1850-1856.

[10] Engelberger RP1, Kucher N.Ultrasound-assisted thrombolysis for acute pulmonary embolism: a systematic review.Eur Heart J. 2014 Mar;35(12):758-64. 